\title{
Correlation matrix methods for excited meson form factors in Full QCD
}

\section{Benjamin Owen ${ }^{* a}$, Waseem Kamleh ${ }^{a}$, Derek Leinweber ${ }^{a}$, Selim Mahbub ${ }^{a}$ and Benjamin Menadue $^{a}$}

${ }^{a}$ Special Research Centre for the Subatomic Structure of Matter (CSSM),

School of Chemistry and Physics, University of Adelaide, SA 5005, Australia

E-mail: benjamin.owendadelaide.edu.au

In recent years, there has been increasing interest in hadronic excitations. So how would one go about exploring hadron resonances and their structure in the context of Lattice QCD? We outline how the variational method can be utilised for form factor calculations. This variational approach not only provides us with a method for extracting excited state hadron form factors, but also results in dramatic improvement in ground state calculations through the removal of excited state contaminations. Here we present both these aspects in the context of $\pi$ and $\rho$ meson form factors.

The 30th International Symposium on Lattice Field Theory

June 24 - 29, 2012

Cairns, Australia

\footnotetext{
${ }^{*}$ Speaker.
} 


\section{Introduction}

Understanding how the non-trivial structure of hadrons results from QCD has been one of the fundamental goals of Lattice QCD since its inception, but it must be said that this is no easy task. Over the past thirty years, a great deal of progress has been made in exploring the light, charmed and heavy quark sectors. Almost all of these studies have been limited to studying only the ground states in each channel. In recent years there has been increased interest experimentally in nucleon resonances, and so it is worth asking how we would go about exploring the structure of hadronic resonances in the context of Lattice QCD.

Over the past decade, numerous techniques have been developed for examining the excited state spectrum of both baryons and mesons. Of these techniques, the most popular and successful is the variational approach. The underlying principle of this method is to construct an ideal set of interpolators, which couple to a single state in the spectrum, from a pre-existing basis of operators. By isolating individual states, we are able to apply the same techniques used in the extraction of hadronic properties of the ground state to all eigenstates of the Hamiltonian, providing us with a mechanism to probe and investigate the excited state structure directly from QCD.

Though we are still along way off from precision calculations, the resulting spectrum is remarkably similar and as diverse as the observed physical hadron spectrum. Through the variational approach we have been able to tackle some of the long-standing issues regarding the ordering of excitations such as the Roper resonance [ए]] and the $\Lambda(1405)$ []]. Furthermore, this approach has allowed us to explore the QCD spectrum for exotic states that have been proposed, but not yet observed physically.

Having developed a method for isolation of exited states, naturally the next step is to probe its structure. In this work we present a prescription for extracting form factors for excited states by utilising the operators constructed through the variational procedure. We note that similar methods have been presented in reference [B], for charmonium transitions, and in reference [四], then applied specifically to $B^{*} \rightarrow B \pi$ transitions in reference [可]. Here we will, for the first time, present matrix elements for an excited state.

\section{Correlation Matrix methods for matrix elements}

The underlying principle of the variational approach is to construct an ideal set of interpolators which couple dominantly to a single state in the spectrum, from a pre-existing basis of operators $\left\{\chi_{i}(x) \mid i=1, \ldots, N\right\}$. This can be achieved by constructing a matrix of cross-correlation functions from which the different superpositions of excited state contributions are linearly combined to isolate the energy eigenstates. More precisely, due to the discrete nature of the lattice, we can decompose any two-point function into a discrete sum of energy eigenstates

$$
G_{i j}(\vec{p}, t)=\sum_{\alpha} \frac{e^{-E_{\alpha}(\vec{p}) t}}{2 E_{\alpha}(\vec{p})} Z_{i}^{\alpha}(\vec{p}) Z_{j}^{\alpha \dagger}(\vec{p}),
$$

where the parameters $Z_{i}^{\alpha}(\vec{p})=\left\langle\Omega\left|\chi_{i}(0)\right| \alpha, p\right\rangle$ are the coupling strengths. By constructing new operators to be linear combinations

$$
\phi^{\alpha}(x)=\sum_{i} v_{i}^{\alpha} \chi_{i}(x) ; \phi^{\alpha \dagger}(x)=\sum_{j} u_{j}^{\alpha} \chi_{j}^{\dagger}(x),
$$


and a suitable choice of coefficients $v_{i}^{\alpha}$ and $u_{j}^{\alpha}$, our interpolators can couple with a single energy eigenstate

$$
\left\langle\Omega\left|\phi^{\beta}(0)\right| \alpha, p\right\rangle=\frac{\delta^{\alpha \beta} \mathscr{Z}_{\alpha}(p)}{\sqrt{2 E_{\alpha}(\vec{p})}} .
$$

From Eqs. ([2. لل and (‥3) we find that the necessary values for $v_{i}^{\alpha}$ and $u_{j}^{\alpha}$ are the solutions of the following generalised eigenvalue equations

$$
\begin{aligned}
& v_{i}^{\alpha}(\vec{p})\left[G\left(\vec{p}, t_{0}+\Delta t\right)\left(G\left(\vec{p}, t_{0}\right)\right)^{-1}\right]_{i j}=c^{\alpha} v_{j}^{\alpha}(\vec{p}), \\
& {\left[\left(G\left(\vec{p}, t_{0}\right)\right)^{-1} G\left(\vec{p}, t_{0}+\Delta t\right)\right]_{i j} u_{j}^{\alpha}(\vec{p})=c^{\alpha} u_{i}^{\alpha}(\vec{p}),}
\end{aligned}
$$

where the eigenvalue $c^{\alpha}=e^{-m_{\alpha} \Delta t}$.

It is important to note that both Eqs. ([2.4) and (2.5) are evaluated for a given momentum $\vec{p}$ and so the diagonalisation condition is only satisfied when we project with the relevant coefficients as follows

$$
v_{i}^{\alpha}(\vec{p}) G_{i j}(\vec{p}, t) u_{j}^{\beta}(\vec{p}) \propto \delta^{\alpha \beta} .
$$

Thus the two-point correlation function for the state $|\alpha, p\rangle$ is

$$
G^{\alpha}(\vec{p}, t) \equiv v_{i}^{\alpha}(\vec{p}) G_{i j}(\vec{p}, t) u_{j}^{\alpha}(\vec{p}) .
$$

To understand how we can utilise the variational method in form factor calculations, we firstly identify the terms present in the three-point correlation function $G_{i j}^{\mu}\left(\vec{p}^{\prime}, \vec{p} ; t_{2}, t_{1}\right)$. Working from the operator definition, one can show that we require three terms - the vertex amplitude, $\left\langle\beta, p^{\prime}\left|J^{\mu}(0)\right| \alpha, p\right\rangle$, and the coupling terms $\left\langle\Omega\left|\chi_{i}(0)\right| \beta, p^{\prime}\right\rangle$ and $\left\langle\alpha, p\left|\chi_{j}^{\dagger}(0)\right| \Omega\right\rangle$.

$$
G_{i j}^{\mu}\left(\vec{p}^{\prime}, \vec{p} ; t_{2}, t_{1}\right)=\sum_{\alpha, \beta} \frac{e^{-E_{\beta}\left(\vec{p}^{\prime}\right)\left(t_{2}-t_{1}\right)} e^{-E_{\alpha}(\vec{p}) t_{1}}}{2 \sqrt{E_{\alpha}(\vec{p}) E_{\beta}\left(\vec{p}^{\prime}\right)}} Z_{i}^{\beta}\left(\vec{p}^{\prime}\right) Z_{j}^{\alpha \dagger}(\vec{p})\left\langle\beta, p^{\prime}\left|J^{\mu}(0)\right| \alpha, p\right\rangle .
$$

The coupling parameters take the same form as they did in the calculation of two-point function with two key differences. The inclusion of a current means that the initial and final momenta need not be the same, and there also exists the possibility that the initial and final energy eigenstates are not the same. That is, the current can induce a transition between states. With this in mind, to calculate the transition matrix element for radiative transitions between eigenstates $\alpha \rightarrow \alpha^{\prime}$, we require

$$
\left(G^{\alpha \rightarrow \alpha^{\prime}}\right)^{\mu}\left(\vec{p}^{\prime}, \vec{p} ; t_{2}, t_{1}\right)=v_{i}^{\alpha^{\prime}}\left(\vec{p}^{\prime}\right) G_{i j}^{\mu}\left(\vec{p}^{\prime}, \vec{p} ; t_{2}, t_{1}\right) u_{j}^{\alpha}(\vec{p})
$$

while for matrix elements of the state $\alpha$,

$$
\left(G^{\alpha}\right)^{\mu}\left(\vec{p}^{\prime}, \vec{p} ; t_{2}, t_{1}\right)=v_{i}^{\alpha}\left(\vec{p}^{\prime}\right) G_{i j}^{\mu}\left(\vec{p}^{\prime}, \vec{p} ; t_{2}, t_{1}\right) u_{j}^{\alpha}(\vec{p}) .
$$

To isolate the matrix element from the three-point function, we construct the ratio in the usual way. In this work we shall use the ratio defined in reference [ 6 . For the state $\alpha$ the necessary ratio is,

$$
\left(R^{\alpha}\right)^{\mu}\left(\vec{p}^{\prime}, \vec{p}\right)=\sqrt{\frac{\left(G^{\alpha}\right)^{\mu}\left(\vec{p}^{\prime}, \vec{p} ; t_{2}, t_{1}\right)\left(G^{\alpha}\right)^{\mu}\left(\vec{p}, \vec{p}^{\prime} ; t_{2}, t_{1}\right)}{G^{\alpha}\left(\vec{p}, t_{2}\right) G^{\alpha}\left(\vec{p}^{\prime}, t_{2}\right)}} .
$$




\section{Calculation Details}

For this calculation we make use of the PACS-CS $2+1$ flavour dynamical-QCD gauge-field configurations $[\square]$ made available through the ILDG $[\mathbb{8}]$. These configurations are generated using a non-perturbatively $\mathscr{O}(a)$-improved Wilson-Clover fermion action and Iwasaki gauge-action. The lattice volume is $32^{3} \times 64$, with $\beta=1.90$ resulting in a lattice spacing $a=0.0907 \mathrm{fm}$. Of the five available quark masses we will only consider form factors for the heaviest herein, with hopping parameter $\kappa_{u d}=0.13700$ corresponding to a pion mass of $m_{\pi}=0.624 \mathrm{GeV}$. We consider 350 configurations. Our error analysis is performed using a second-order jackknife method, with the $\chi^{2}$ per degree of freedom $\left(\chi_{\text {dof }}^{2}\right)$ obtained via a covariance matrix analysis.

The key to success with the variational approach is to utilise a basis of operators in which there is diversity in the overlap with various excited states. As there are a limited number of local bilinear operators for given $J^{P C}$, a great deal of work has been made by various groups in increasing the available operators. Here we choose to use fermion source and sink smearing as a method of extending our operator basis as outlined in [Q, [0]. In particular we use $n$ sweeps of gauge invariant Gaussian smearing in the spatial dimensions only with smearing fraction $\alpha=0.7$ [Q]. Here we consider four levels of smearing, these being 16,35, 100 and 200, allowing for the construction of up to a $4 \times 4$ correlation matrix. For the pion our local interpolator is $\chi_{\pi}(x)=\bar{d}(x) \gamma_{5} u(x)$, and for the rho meson $\chi_{\rho}^{i}(x)=\bar{d}(x) \gamma^{i} u(x)$. For the vartiaonal analysis we use $t_{0}=17$ and $\Delta t=2$ relative to the source at $t=16$. In Fig. 1 we present our results from the variational analysis for the pi and rho ground states and their first excitations at the three heaviest quark masses. The ground state masses for the remaining quark masses are also presented.

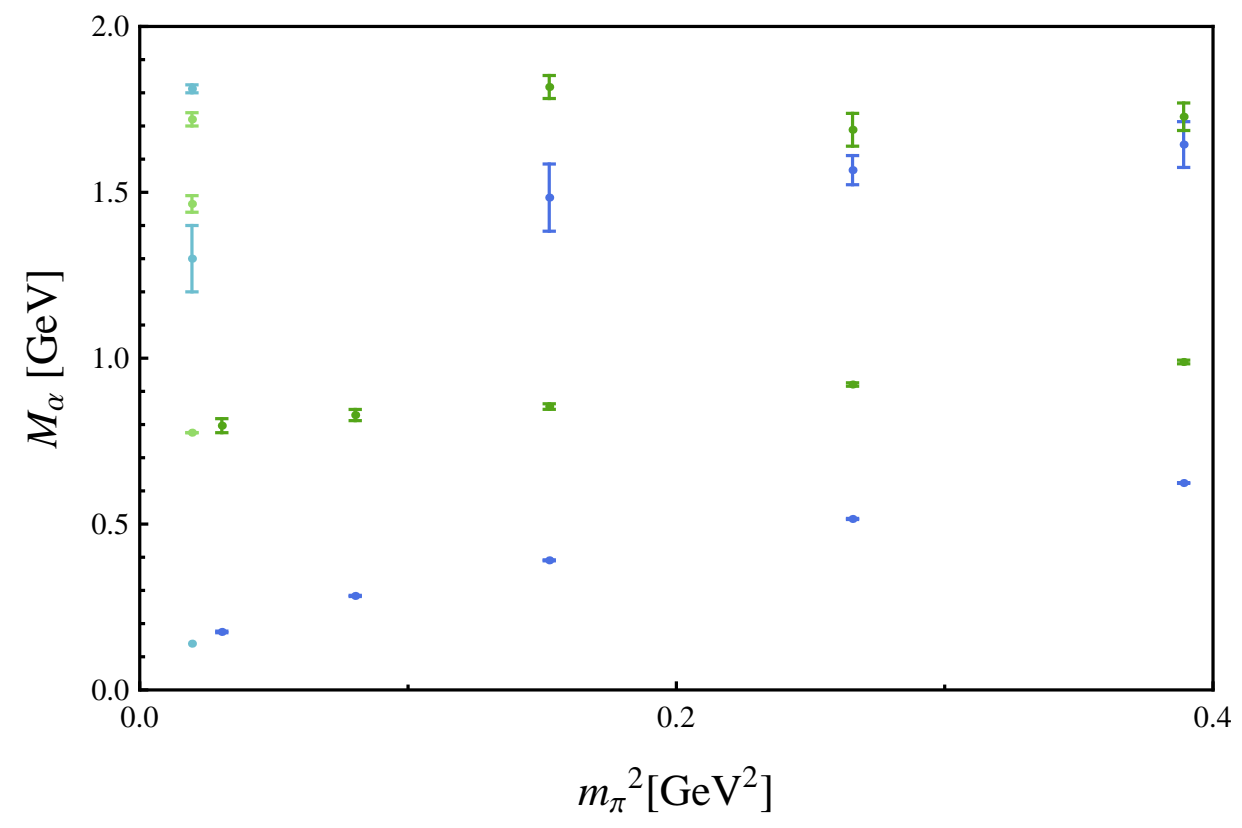

Figure 1: Meson spectrum from the PACS-CS configurations. We have performed a correlation matrix analysis at the three heaviest quark masses and plot the ground-state and first excitation pion $\left(0^{-}\right)$(blue), rho meson $\left(1^{-}\right)$(green). The points at the far left correspond to the states in the PDG summary tables. 
To extract the pseudoscalar and vector meson form factors, we follow the prescription outlined by Hedditch et al. [焑]. Here we will simply identify the expressions used to calculate the form factors from the ratio of Eq. (R,IO) and refer the reader to reference [目] for details. For the pion, only a single form factor is required, which when identified as a Sachs form factor corresponds to the charge form factor, $G_{C}\left(Q^{2}\right)$,

$$
G_{C}\left(Q^{2}\right)=2 \frac{\sqrt{E_{\pi} m_{\pi}}}{E_{\pi}+m_{\pi}} R^{0}\left(p^{\prime}, p\right)
$$

For the rho meson, a further two terms are required due to its non-trivial spin. When expressed in terms of Sachs form factors, these correspond to the charge form factor, $G_{C}\left(Q^{2}\right)$, the magnetic form factor, $G_{M}\left(Q^{2}\right)$, and the quadrupole form factor, $G_{Q}\left(Q^{2}\right)$. In this calculation we access these using the following expressions [6],

$$
\begin{aligned}
G_{C}\left(Q^{2}\right) & =\frac{2}{3} \frac{\sqrt{E_{\rho} m_{\rho}}}{E_{\rho}+m_{\rho}}\left(R_{1}{ }^{0}{ }_{1}+R_{2}{ }^{0}{ }_{2}+R_{3}{ }^{0}{ }_{3}\right), \\
G_{M}\left(Q^{2}\right) & =\frac{\sqrt{E_{\rho} m_{\rho}}}{p_{x}}\left(R_{1}{ }^{3}{ }_{3}+R_{3}{ }^{3}{ }_{1}\right), \\
G_{Q}\left(Q^{2}\right) & =m_{\rho} \frac{\sqrt{E_{\rho} m_{\rho}}}{p_{x}^{2}}\left(2 R_{1}{ }^{0}{ }_{1}-R_{2}{ }^{0}{ }_{2}-R_{3}{ }^{0}{ }_{3}\right) .
\end{aligned}
$$

This analysis is performed upon the ground-states and their first excitation at the heaviest quark mass using the projected correlation functions obtained through Eqs. (2.7) and (2.9). To provide a bench mark and allow for comparison with the standard approach, we also perform the calculation using an ensemble of correlation functions with a given smeared source. In this case we choose 35 sweeps of source smearing, and a point sink as representative of the standard approach.

\section{Ground-state Form Factors}

In Fig. 2 we present the results for the ground state form factors. The blue-circular data points correspond to our improved variational method while the red-square data points correspond to the standard, single correlation function method. In the upper two plots, which correspond to the pion and rho meson charge form factors respectively, we can see that both methods produce nice clean signals with distinct plateaus. In both plots the presence of excited state effects after the insertion of the current is apparent, but the almost step-like behaviour in the case of the variational method indicates that these effects are significantly smaller. By using operators that are optimised to couple with the ground-state, near-by excited states have been suppressed resulting in improved ground state dominance and earlier fit windows. In the case of the pion's $G_{C}$, based upon the $\chi_{\mathrm{dof}}^{2}$, the earliest time slice one can fit would be $t_{S}=30$ for the standard approach while in the case of the variational method one can begin fitting as early as $t_{S}=25$. Similar conclusions can be drawn for the fits of the rho meson $G_{C}$.

Where our variational approach really pays off is in the more difficult channels of the rho meson. The improvement exhibited for the rho meson magnetic and quadrupole form factors is substantial. Using the standard approach for $G_{M}$, due to an almost linear drift in the result, a discernible plateau is not observed until atleast 6-7 time slices after the insertion of the current. In 

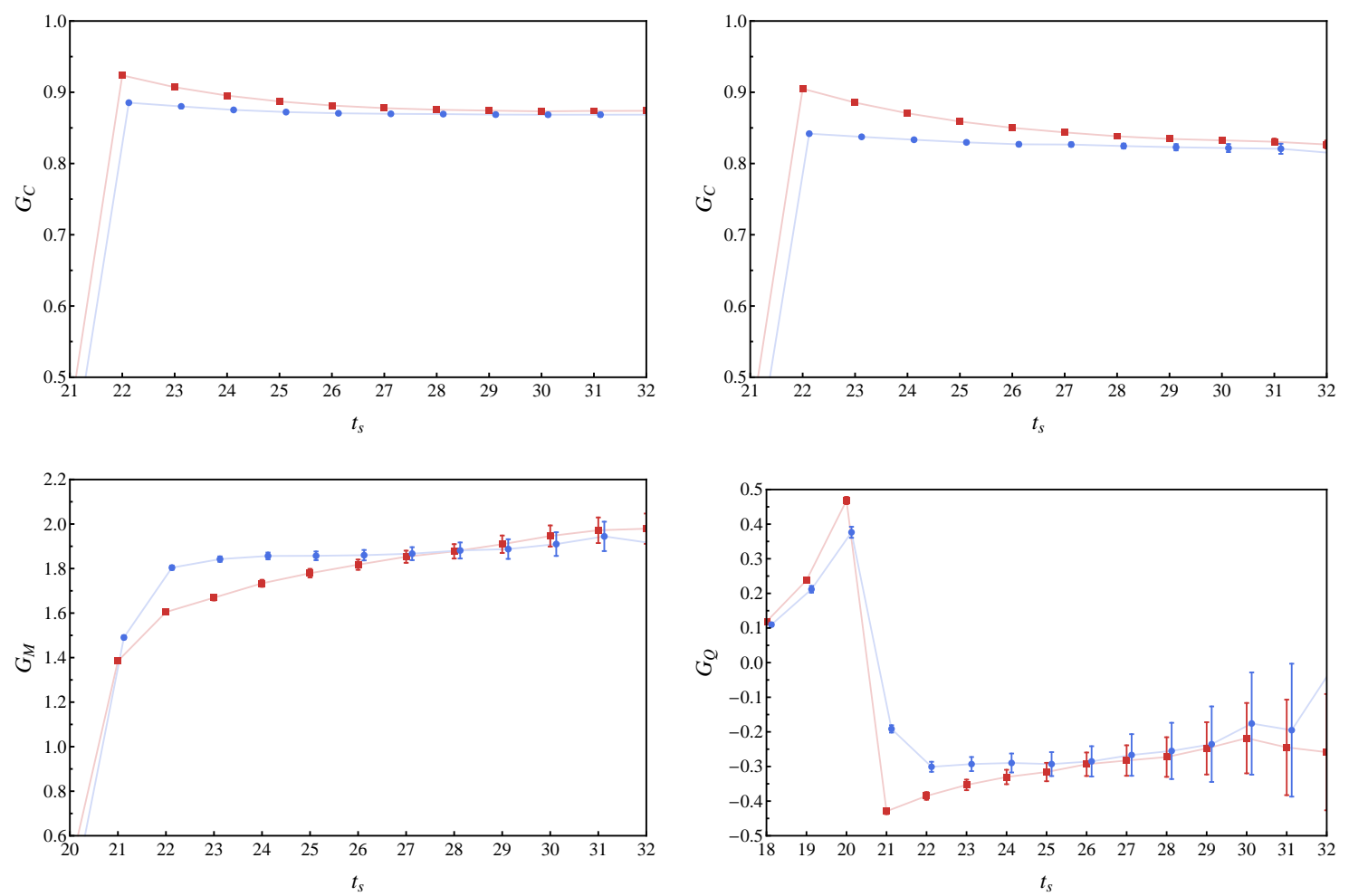

Figure 2: Comparison of the standard, single correlation function approach (red-square symbols) with our improved variational approach (blue-circle symbols). Top left is the pion $G_{C}$, top right is the rho meson $G_{C}$, bottom left is the rho meson $G_{M}$ and bottom right is the rho meson $G_{Q}$. In all cases we find an early onset of ground state dominance and in the case of the more difficult magnetic and quadrupole form factors, the quality of the plateau has dramatically improved.

contrast to this, our variational approach provides a rapid onset of the plateau. A distinct plateau is observed within 2-3 time slices after the point-split current insertion centred at $t=21$. This is clear evidence that through the use of optimised operators obtained from a variational procedure one is able to systematically reduce the impact of excited state effects. Similar behaviour is observed for $G_{Q}$.

\section{Excited State Form Factors}

The most intriguing aspect of using a variational approach is that it opens up a new realm of interesting physics. As the excited states exhibit the same quantum numbers as their groundstate counterparts, the same expressions can be considered using the relevant projected correlation functions $G^{\alpha}\left(p, p^{\prime}\right)$ in the analysis. In Fig. 3 we present the worlds first results for the form factors of an excited state hadron, in this case the first rho meson excitation. We note that similar results are obtained for the first excitation of the pion. As outlined in reference [目] one can infer rms-charge radii and magnetic moments for these states from $G_{C}\left(Q^{2}\right)$ and $G_{M}\left(Q^{2}\right)$ respectively ${ }^{1}$. In Table 1

\footnotetext{
${ }^{1}$ This approach adopts a monopole ansatz for the $Q^{2}$ dependence of the charge form factor. While the multi-particle Fock-space component of the excited state certainly give rise to a more interesting $Q^{2}$ dependence, the ansatz does provide some insight into the nature of this excited state
} 

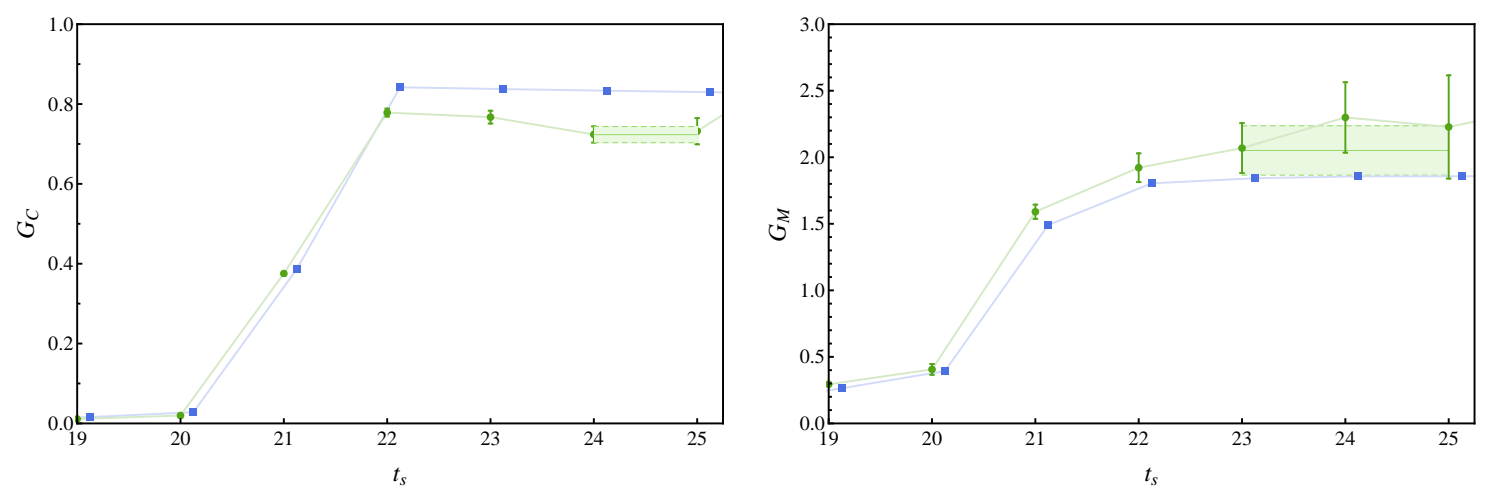

Figure 3: The charge and magnetic form factors for the first excitation of the rho meson (greencircle symbols). The shaded region is the best fit to the data in the region where there is a clear signal. The blue-square symbols illustrate the ground-state result for comparison.

we display our results for the ground state and first excitation in each channel. The data indicates that these excitations are more extended than their ground-state counterparts.

\begin{tabular}{c||c|c||c|c}
\hline \multicolumn{1}{l||}{} & \multicolumn{2}{c||}{$r_{r m s}[\mathrm{fm}]$} & \multicolumn{2}{c}{$\mu\left[\mu_{N}\right]$} \\
\hline & Ground-state & $1^{\text {st }}$ excitation & Ground-state & $1^{\text {st }}$ excitation \\
\hline$\pi\left(0^{-}\right)$ & $0.51 \pm 0.01$ & $0.73 \pm 0.09$ & - & - \\
\hline$\rho\left(1^{-}\right)$ & $0.59 \pm 0.01$ & $0.79 \pm 0.04$ & $2.09 \pm 0.02$ & $2.7 \pm 0.3$ \\
\hline
\end{tabular}

\section{Acknowledgements}

This research was undertaken with the assistance of resources at the NCI National Facility in Canberra, Australia, the iVEC facilities at Murdoch University (iVEC@ Murdoch) and the University of Western Australia (iVEC@UWA). These resources were provided through the National Computational Merit Allocation Scheme, supported by the Australian Government. This research is supported by the Australian Research Council.

\section{References}

[1] M. S. Mahbub et al. [CSSM Lattice Collaboration], Roper Resonance in 2+1 Flavor QCD, Phys. Lett. B 707, 389 (2012) [arXiv: 1011.5724 [hep-lat]].

[2] B. J. Menadue, W. Kamleh, D. B. Leinweber and M. S. Mahbub, Isolating the $\Lambda(1405)$ in Lattice QCD, Phys. Rev. Lett. 108, 112001 (2012) [arXiv:1109.6716 [hep-lat] ].

[3] J. J. Dudek, R. Edwards and C. E. Thomas, Exotic and excited-state radiative transitions in charmonium from lattice QCD, Phys. Rev. D 79, 094504 (2009) [arXiv: 0902.2241 [hep-ph] ].

[4] J. Bulava, M. Donnellan and R. Sommer, On the computation of hadron-to-hadron transition matrix elements in lattice QCD, JHEP 1201, 140 (2012) [arXiv:1108.3774 [hep-lat] ].

[5] F. Bernardoni, B. Blossier, J. Bulava, M. Della Morte, P. Fritzsch, N. Garron, A. Gerardin and J. Heitger et al., B-physics from HQET in two-flavour lattice QCD, [arXiv:1210.7932 [hep-lat] ]. 
[6] J. N. Hedditch, W. Kamleh, B. G. Lasscock, D. B. Leinweber, A. G. Williams and J. M. Zanotti, Pseudoscalar and vector meson form-factors from lattice QCD, Phys. Rev. D 75, 094504 (2007) [hep-lat/0703014].

[7] S. Aoki et al. [PACS-CS Collaboration], 2+1 Flavor Lattice QCD toward the Physical Point, Phys. Rev. D 79, 034503 (2009) [arXiv: 0807.1661 [hep-lat] ].

[8] M. G. Beckett, B. Joo, C. M. Maynard, D. Pleiter, O. Tatebe and T. Yoshie, Building the International Lattice Data Grid, Comput. Phys. Commun. 182, 1208 (2011) [arXiv: 0910.1692 [hep-lat]].

[9] M. S. Mahbub, A. O.Cais, W. Kamleh, B. G. Lasscock, D. B. Leinweber and A. G. Williams, Isolating Excited States of the Nucleon in Lattice QCD, Phys. Rev. D 80, 054507 (2009) [arXiv:0905.3616 [hep-lat]].

[10] M. S. Mahbub, A. O. Cais, W. Kamleh, D. B. Leinweber and A. G. Williams, Positive-parity Excited-states of the Nucleon in Quenched Lattice QCD, Phys. Rev. D 82, 094504 (2010) [arXiv:1004.5455 [hep-lat]]. 